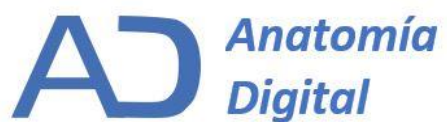

\section{Gimnasia musical aerobia}

\author{
Aerobic music gymnastics
}

Lic. José Días Valdez. ${ }^{1}$ \& Msc. Eugenio Rodolfo González Pérez. ${ }^{2}$

Recibido: 08-09-2017 / Revisado: 12-09-2017 / Aceptado: 20-20-17 / Publicado: 04-10-2017

DOI: https://doi.org/10.32/cienciadigital.v3i1.947

Abstract.

A comprehensive search was conducted on the $\mathrm{u}$

Keywords: Reflexology

\section{Resumen.}

Se realizó una búsqueda exhaustiva del uso del ejercicio físico aplicado al Adulto Mayor.

Palabras claves: Reflexología

\section{Introducción.}

Divertida, desenfadada y fácil de practicar, la danza aeróbica se empezó a difundir por muy diversos países, gracias, en parte, a la aportación de algunas personas famosas como Jane Fonda o Sidney Rome, que, tras descubrir el sentido lúdico de este tipo de ejercicio, pero también sus extraordinarios efectos para el organismo, decidieron contribuir en la tarea de darlo a conocer.

Los orígenes del aerobic tal y como lo entendemos en la actualidad, podemos situarlos en el año 1968. En este año apareció publicado por primera vez en EEUU un libro titulado "aerobics". Su autor el Doctor Kenneth H. Cooper, medico de las fuerzas armadas estadounidenses, expone en la obra, el programa de entrenamiento que él mismo diseñó para los miembros de las fuerzas armadas de su país. Sin embargo, algunas fuentes atribuyen el término "aerobic" a Pasteur (Francia 1875)

\footnotetext{
${ }^{1}$ Facultad de Ciencias Médicas de Mayabeque, Mayabeque, Cuba, jdiaz@infomed.sld.cu

${ }^{2}$ Facultad de Ciencias Médicas de Mayabeque, Mayabeque, Cuba, egonzalez@infomed.sld.cu
} 
El programa del Dr. Kenneth consistía en llevar a cabo esfuerzos durante periodo de tiempo prolongado, con el fin de aumentar el rendimiento y la resistencia de quienes lo realizaban, disminuyendo así el porcentaje de riesgo a sufrir enfermedades cardíacas y respiratorias (infarto, arterioesclerosis). En este primer tratado sobre el aerobic, se predican las excelencias del ejercicio aeróbico y se defiende la práctica de un ejercicio físico de baja y mediana intensidad, cuyo objetivo fundamental es el desarrollo del sistema cardiovascular. Define en su primer libro al entrenamiento aeróbico como una actividad que se puede realizar durante largo tiempo debido al equilibrio existente entre el suministro y consumo de oxígeno que el organismo necesita para la producción de energía.

En 1969 Jackie Sorensen propone a Kenneth H. Cooper la posibilidad de utilizar la danza aeróbica como método de entrenamiento gimnástico para las esposas de los militares norteamericanos en una base de Puerto Rico frente a la tradicional gimnasia (de mantenimiento). Tras el éxito de "aerobics" Kenneth publicó en 1970 un segundo tratado sobre el aerobic adaptado a personas mayores de 35 años titulado "The new aerobics" y un tercero adaptado especialmente para mujeres titulado "aerobics for Women". Jackie Sorensen funda ese mismo año en New Jersey el "aerobic Dancing inc." primer estudio donde se ofrecen clases de aerobic al público en general.

A partir de este programa inicial, creado por el que se considera el padre del aerobic, apareció primero en EEUU y posteriormente en otros países del mundo la moda del jogging, que es la forma más popular de practicar un entrenamiento aeróbico de resistencia.

Con el paso de los años se pensó en la posibilidad de combinar música y elementos de disciplinas diferentes: jogging, jazz, gimnasia, baile, y crear así algo nuevo. El resultado de esta combinación es lo que se ha denominado danza aeróbica (aerobic Dance), que consiste en bailar al ritmo de la música, pero de un amanera aeróbica, es decir, siguiendo los principios básicos que debe de cumplir todo ejercicio aeróbico.

A principios de los 80 el aerobic Dance llega a Europa donde desde 1969 se practicaba la Danza Jazz sobre la que más tarde Judi Sheppard fundaría una modalidad denominada Jazzercise. Así mismo Monika Becman había creado la Gimnasia Jazz con base en la gimnasia moderna y nacida por la necesidad de utilizar la música cómo factor educativo en sus clases de educación física.

Pero, aunque a simple vista este tipo de terapias pueden parecer iguales al aerobic, en muchos estudios se ha tratado erróneamente de atribuirles la paternidad del aerobic. Si atendemos a las bases sobre las que se han fundado este tipo de estudios, sería como afirmar que el Skateboard tiene su origen en el patinaje tradicional. Indudablemente tanto Roller como Skaters utilizan ruedas y se basan en deslizarse sobre ellas, pero el origen del Skateboard proviene de la necesidad de hacer surf o wind surf en sitios donde no hay mar ni olas o viento. De esta manera, aunque el resto de terapias utilicen música y se basen en la realización de un 
ejercicio físico, sólo el aerobic contiene un objetivo básico e indispensable que es el hecho de realizar un entrenamiento gimnástico seguro y eficaz, utilizando únicamente la energía proveniente del sistema aeróbico de producción de energía. Aunque en el aerobic Dance se utilice la música y se baile con ella su origen no proviene del baile, sino del entrenamiento de ejercicios aeróbicos.

Hoy día la danza aeróbica posee innumerables seguidores en todo el mundo. En 1988 el aerobic era el tercer deporte más practicado en los EEUU pasando a ocupar el segundo lugar un año después.

Multitud de practicantes se están beneficiando desde hace algunos años de este ejercicio tan saludable y completo, ya que en él no sólo se trabaja la resistencia, sino que además se potencia la flexibilidad, la coordinación, la fuerza e incluso la habilidad. Sus beneficios rebasan el campo físico. Se ha constatado la gran mejoría que experimenta el estado anímico del practicante de aerobic, que gana seguridad en sí mismo, ve como mejoran sus relaciones humanas y vence sus complejos con mayor facilidad. Médicamente, se ha comprobado una mejora integral de los ancianos, de la embarazadas, de los convalecientes, de disminuidos psíquicos, etc.

El poder abandonarse al ritmo de la música siguiendo toda una serie de variados pasos, siempre adaptados a las condiciones individuales de cada uno, hace las delicias de quienes los practican. Sin embargo, para que el entrenamiento sea beneficioso, efectivo y seguro será necesario haber aprendido la técnica correcta y propia que tiene el aerobic. Dependiendo de la condición física, de las características corporales, de posibles enfermedades que se sufran, etc., las actividades que para unas personas resultan aeróbicas porque estimulan el sistema cardiovascular hasta hacerle alcanzar el nivel de entrenamiento idóneo, para otras no lo son.

Por ejemplo, para personas con problemas algo serios de obesidad, el caminar a una marcha ligera y regular puede ser un ejercicio aeróbico muy adecuado, mientras que para una persona sin ese problema y bien entrenada no será suficiente. Esta última necesitará una actividad más intensa ya que por el contrario no conseguirá ejercitarse a su nivel de entrenamiento.

El tiempo es un factor que también contribuye en el hecho de convertir una determinada actividad en un ejercicio aeróbico.

Pero no debemos contemplar al aerobic Dance como único ejercicio aerobic. Existen un número de actividades deportivas que pueden ser aeróbicas si re realizan durante un periodo relativamente largo y a una intensidad moderada.

\section{Objetivo general:}

Sustentar la práctica de la Gimnasia Aerobia c como un método de prevención y tratamiento contra el fenómeno social del sedentarismo. 


\section{Desarrollo}

El término aerobic significa literalmente: "Con Oxígeno" (O2). Pero para entender bien este planteamiento, deberemos ahondar primero en determinados principios básicos sobre la producción de energía en nuestro organismo. Energía, es simplemente la habilidad para realizar un trabajo. La contracción de los músculos que nos permiten caminar o movernos, el crecimiento de tejido nuevo en los niños e incluso en los adultos durante la recuperación de una lesión son algunas de las distintas formas de trabajo físico o biológico que requieren energía. Pero vayamos un poco más lejos y descubramos de donde viene toda esa energía que necesitamos para desarrollar el trabajo.

¿Crees tú que la energía que estás utilizando en la sala mientras practicas aerobics proviene del sol?

Pues así es.

La energía que estamos utilizando para contraer nuestros músculos tiene su origen en el sol; pero no es posible que absorbas energía mientras estás haciendo ejercicio bajo el sol. Esta energía solar necesitará transformarse de energía luminosa a una forma de energía química que el cuerpo pueda utilizar. La transformación de esta energía luminosa se inicia cuando las plantas verdes absorben ésta a través del proceso de fotosíntesis. Las plantas inician esta cadena produciendo compuestos sintéticos muy simples, tales como el agua y el dióxido de carbono; y en presencia de la luz, moléculas alimenticias complejas que contienen una gran cantidad de energía química almacenadas en forma de glucosa, carbohidratos, grasas y proteínas. Los humanos y los animales pueden obtener energía mediante la ingestión de estas plantas utilizándolas, así como fuente de combustible.

A lo largo de la ruta que sigue la energía solar para llegar al humano no ocurre ninguna creación de energía. Ni lo humanos ni las plantas pueden crear energía. Lo único que ocurre es una transformación. Los humanos, después de haber ingerido a las plantas, las transforman nuevamente en energía. Al llegar a este punto, la energía puede ser utilizada para el trabajo biológico o almacenarse para un uso posterior. Los principales lugares de almacenamiento, son: el tejido adiposo, el músculo esquelético y el hígado. Pero ninguna de estas transformaciones resultará $100 \%$ eficaz. De hecho, los humanos, usamos o almacenamos, menos de la mitad de la energía disponible en la comida que ingerimos. La energía no utilizada o perdida escapa transformada en calor. La siguiente ecuación expresa la relación o el balance entre la energía que entra en el cuerpo y la que se usa, se almacena, y se pierde.

Energía entrante $=$ Energía usada + Energía almacenada + Energía perdida .

Antes de poder usarse, la energía debe convertirse primero en un compuesto llamado Adenosintrifosfato (ATP). El hecho de que el ATP posea gran cantidad de energía, se debe 
en gran parte a la manera que está estructurado. El último grupo de fosfato, se adhiere al sobrante de la molécula por medio de una unión de "alta energía". Cuando esta unión se rompe el grupo de fosfato se libera, y al mismo tiempo una cantidad sustanciosa de energía. El resultado final es el Adenosindifosfato (ADP) y fosfato (P). Esta ruptura del ATP es la única fuente de energía para desarrollar la contracción muscular, y toda la energía almacenada en el cuerpo deberá convertirse primero en ATP antes de poder utilizarse durante el ejercicio. Esta reacción producida por el ATP es también una reacción reversible, es decir, puede sintetizarse ATP nuevamente si existe una fuente de ADP, P y energía.

Aun cuando el siglo XXI se inicia entre grandes adelantos tecnológicos y descubrimientos científico y técnicos que se extienden a todos los campos con gran rapidez, el ser humano sigue siendo el mismo y mantiene las necesidades primordiales de seguirse alimentando para conservar la salud y gastando energías para poder acometer las tareas y acciones de la vida diaria. Por eso hoy el mundo no solo se preocupa de cómo alimentar a los hombres, sino también de cómo hacerlo gastar energías mediante actividades físicas que no agredan al organismo humano.

En esta búsqueda muchos concuerdan en que las actividades aeróbicas resultan imprescindibles para resguardar y mantener la salud.

En sus inicios las actividades aeróbicas se limitaban solamente a largas caminatas, trotes o carreras con diferentes ritmos de trabajo, al aire libre, a campo traviesa o por las calles de cualquier ciudad. Luego se desarrollaron algunos aparatos sofisticados para poder trabajar bajo techo y en espacios reducidos entre los que se destacan la bicicleta estacionaria, el remo, las esteras rodantes entre otros. Estos aparatos han tenido un exitoso y comercial camino, pero por no poder encontrarse al alcance de todos y también por mantener las mismas posturas y posiciones iniciales que limitan la gama de movimientos a realizar, es que se recurre nuevamente a las actividades de la gimnasia de mantenimiento, pero esta vez introduciendo como componente novedoso el trabajo aeróbico.

Este trabajo se realiza mediante pasos y sus combinaciones y como componente de importancia se incluye el trabajo con la música, la cual además de motivar, sirve para dosificar adecuadamente las cargas de trabajo de las clases. Es así como surge esta nueva modalidad, denominada Gimnasia Aerobia de Salón.

\section{La clase de gimnasia aeróbica}

Su estructura y contenidos fundamentales.

Rutinas... Formato de una clase

El formato de la clase tiene bases fisiológicas y lo puedes adaptar a tus preferencias personales, la siguiente secuencia es para una clase de una hora: 
- Instrucción previa: En este momento les explicas a tus alumnos el objetivo de tu clase, cómo está estructurada y cómo pueden evitar lesiones, además de otras indicaciones generales.

- Calentamiento

- Fase aeróbica

- Enfriamiento

- Ejercicios de tono muscular

- Relajamiento final

Calentamiento: El objetivo del calentamiento es preparar a tu cuerpo para realizar ejercicios más vigorosos y con más intensidad y así poder reducir al mínimo el riesgo de que sufras una lesión irreparable, así que a tu calentamiento mínimo deberás darle un tiempo de 8 a 12 minutos, y hacer una combinación de movimientos rítmicos y movimientos estáticos involucrando la mayor cantidad de grupos musculares y al realizarlos deberán ser con control y suavidad y así lograrás un estado estable(cuando la demanda y el suministro de oxígeno alcanzan niveles iguales).

Para que tu calentamiento tenga fluidez deberás seguir una secuencia donde incluyas a todos los grupos musculares importantes, a continuación, agregamos una lista que pensamos te será de gran utilidad y con un orden especifico de arriba hacia abajo.

Calentamiento estático: Coloca tu cuerpo en una posición totalmente estática, abre tus piernas a una posición cómoda con las rodillas relajadas con la espalda recta y tu pelvis apuntando hacia abajo, relaja tus hombros y llévalos hacia atrás contrae tu abdomen y tus glúteos.

Calentamiento rítmico: Ahora es momento de empezar a involucrar a tus grupos musculares más grandes con ejercicios rítmicos, como su nombre lo indica son ejercicios multimusculares dónde vas a empezar a mover tu cuerpo con movimientos suaves y controlados. Estos ejercicios te ayudarán a preparar al tu cuerpo para realizar ejercicios más intensos y más vigorosos, ya que te aumentarán el rango movimiento de las articulaciones y sus tejidos aledaños incrementando tu temperatura muscular y tu flujo sanguíneo hacia tus articulaciones, también puedes utilizarlo para preparar tus ejercicios que realizaras más adelante en tu fase aeróbica.

Combinación de los dos calentamientos: La combinación de los dos calentamientos deberás hacerlo de tal manera que tu actividad física sea estable esto quiere decir que no eleves ni bajes demasiado la intensidad, empieza por escoger movimientos que no demanden grandes cantidades de oxígeno y así logres mantenerte estable entre fase y fase de cada calentamiento. 
Vol. 1, N², p. 24-36, octubre - diciembre, 2017

A continuación, te presentamos un ejemplo de cada uno, para lograr una combinación adecuada.

Primera Fase:

Ejercicios rítmicos de flexibilidad en su lugar. Ejemplo: Marcha, paso "v", abrir y cerrar, tocar con punta.

Segunda Fase:

Calentamiento estático de la parte superior: Cuello (esternocleidomastoideo), Hombros(deltoides), Parte media de la espalda(dorsal), Parte alta de la espalda(trapecio), Pechos(pectoral), Espalda baja (zona lumbar), Fibras laterales del abdomen(oblicuos).

Tercera Fase:

Ejercicios rítmicos de flexibilidad con desplazamientos sin elevación de rodillas. Ejemplo: desplazamientos al frente, hacia atrás, desplazamientos laterales.

\section{Cuarta Fase:}

Calentamiento estático de la parte inferior: Muslos (grupo de los cuádriceps, femorales, Izquiotiviales, sartorio), Glúteo (glúteo máximo), Muslo externo(abductor), Muslo interno(aductor), Pantorrilla(gastronemio)

Quinta Fase:

Ejercicios rítmicos de flexibilidad combinando la primera y tercera fase. Ejemplo: Marcha combinado con desplazamiento al frente. Paso "v" combinado con desplazamiento lateral.

Nota: en esta fase ya podrías elevar un poco las piernas para empezar a elevar tu frecuencia cardiaca y prepararte para transición

(Curva de campana) a la fase aeróbica.

Transición a la fase aeróbica:

La transición a la fase aeróbica debe asemejarse a una curva de campana en la gráfica de la intensidad de la fase aeróbica. La transición entre el calentamiento y la fase aeróbica se logra elevando la intensidad. Ejemplo:

- Eleva los brazos

- Muévete más intensamente 


\section{A Anatomia

- Aplaude

Fase aeróbica: (secuencia)

Deberás iniciar tu fase aeróbica con movimientos que vayan incrementando la intensidad, estos deberán ser suaves y controlados. Para lograr un buen control de tu estado estable además de evitar alguna lesión de tus pies y tobillos deberás evitar ejercicios de alta intensidad como son los llamados Jumping Jacks, desplazamientos laterales, péndulos, durante los tres primeros minutos de tu fase aeróbica. Deberás combinar ejercicios de alta intensidad con ejercicios de baja intensidad e intercalarlos de tal manera que el resultado alcance un estado estable.

Es importante que conserves una postura adecuada durante toda la fase aeróbica, esto significa que deberás mantener:

- Tu espalda y abdomen contraído

- Al trotar tus talones deberán llegar siempre al piso

- No debes trotar sobre los dedos de tus pies

- Balancea el peso de tu cuerpo sobre toda la planta de tus pies y no solo sobre los talones

- Evita elevar tus talones hasta tocar tus glúteos ya que este ejercicio hará que tu espalda se arqueé y te cause un estrés

- Es muy importante la respiración durante toda la fase aeróbica, así que deberás hacerla de una manera suave y controlada con forme te lo requieran tus ejercicios.

Variedad de movimientos:

Ahora deberás mantener el interés tanto tuyo cómo para tus alumnos, además de trabajar la mayor cantidad de tus grupos musculares. Es muy importante que vayas construyendo tu clase de tal manera que tus combinaciones no sean tan complicadas, además de poner atención en el nivel de condición que te encuentres o que se encuentren tus alumnos, si tus movimientos y combinaciones requieren coreografía de brazos y piernas al mismo tiempo ve desglosando primero piernas y después brazos gradualmente o viceversa. Evita las patadas elevadas y brincos en un solo pie por largos periodos de tiempo ya que el impacto y las repeticiones del mismo te pueden ocasionar serias lesiones.

Enfriamiento: 
El enfriamiento te ayudara a que tengas una buena transición entre tus ejercicios de la fase aeróbica y tus ejercicios de tono muscular, recuerda que tu corazón está en una etapa de estrés y deberás ayudarlo a regresar a su estado normal y debes hacerlo gradualmente, además que la sangre que fue enviada a las extremidades durante todo el ejercicio aeróbico deberá regresar con eficacia y rapidez al

corazón por lo tanto tu enfriamiento deberá tener un buen tiempo disponible realizando movimientos de brazos y piernas a una intensidad suave y moderada puedes apoyarte de movimientos tipo baile.

\section{Estiramientos:}

Estira tus músculos que involucraste durante tu fase aeróbica, poniendo énfasis en tus (pantorrillas, muslo interno, muslo externo, cuádriceps, flexores de la cadera, isquiotiviales y la espalda baja) deberás estirar cada uno de estos músculos estáticamente antes de terminar tu clase o proseguir con tus ejercicios de tono muscular.

En sus inicios la gimnasia Aerobia, fue diseñada solamente para la población adulta sana, pero hoy en día en nuestro país esta práctica se ha extendido a escolares de la enseñanza media y superior, que lo practican algunos como programas y otros como horarios del deporte participativo.

La resistencia Aerobia o cardio vascular es la capacidad del organismo para realizar esfuerzos de más de tres minutos de duración en equilibrio entre el consumo y suministro de energías a partir de la utilización del oxígeno cuyo suministro se va asegurando por la intervención de los aparatos respiratorios y cardio vasculares.

La resistencia Aerobia permite un mayor tiempo de trabajo a costa de la disminución de la intensidad del mismo.

La Gimnasia Aerobia practicada sistemática y dosificada, mejora la capacidad vital, reduce la frecuencia cardiaca en reposo, aumenta el gasto calórico y las concentraciones de hemoglobina, disminuye el tiempo de recuperación del organismo.

La clase, puede impartirse a la población en instalaciones deportivas, áreas de salud, gimnasios salones de belleza, etc.

La clase, es un proceso concatenado donde se proponen y desarrollan técnicas para el desarrollo de la resistencia Aerobia.

La clase mantiene el siguiente formato.

Tiempo de duración ideal. 1 hora. 
Partes de la clase:

Parte preparatoria.

Dura entre 10 y 12 minutos.

Es donde se forma el grupo se informa brevemente los objetivos de la clase, (esta clase tiene como objetivo principal el desarrollo de la resistencia Aerobia) y se realiza la primera toma de pulso.

Posteriormente se pasa al calentamiento, donde se realizan ejercicios de fácil ejecución y bajos en carga, que permitan preparar al organismo para enfrentar las tareas de la parte principal de la clase. Se incluyen estiramientos estáticos, círculos de las articulaciones, flexiones y extensiones de estas,

Parte Principal.

Denominada también Cardio Vascular. Es la parte más importante de la clase, en ella se trabajan los ejercicios destinados al desarrollo de la resistencia, esta se inicia con la segunda toma de pulso.

El tiempo de duración de esta parte oscila entre los 40 y 45 minutos, de los cuales el $80 \%$ se destina a la resistencia y el otro $20 \%$ a la gimnasia localizada si es que esta forma parte de los objetivos de la clase, si no puede dedicarse todo este tiempo a los aeróbicos.

Esta parte del desarrollo de la resistencia, puede hacerse libre, o por coreografías. Toda esta parte Aeróbica se trabaja de pie con ejercicios de locomoción, desplazándose en diferentes direcciones y usando intensidad de trabajo moderada que permita a quienes la están practicando no llegar a la sofocación o falta de aire.

Los movimientos que más se utilizan en esta parte son los pasos básicos, los auxiliares y sus combinaciones, también movimientos de iguales características a estos, pero creado por los profesores.

Es aquí donde se aplican los ejercicios de bajo y alto impacto.

El bajo impacto, cuando trabajamos siempre con uno de los dos pues en contacto con el piso y cuando las pulsaciones se elevan entre un 40 y un $60 \%$ del pulso de reposo.

Los ejercicios de Alto impacto, son aquellos que se realizan con pequeños saltos, estos elevan las pulsaciones de reposo entre un 60 y un $85 \%$ del pulso de reposo.

Esta parte de la clase puede desarrollarse tradicionalmente utilizado el procedimiento frontal, o también usando los circuitos aeróbicos, y los intervalos de trabajo. 
La música que se utiliza debe estar acorde con los objetivos propuestos en esta parte, siempre debe estar editada, sin espacios en blanco, buscando que nos ayude la misma a orientar el trabajo adecuadamente.

Al finalizar la parte aeróbica, se realiza la tercera toma de pulso, posteriormente se realizan algunos ejercicios de recuperación y respiración, para bajar el pulso y poder pasar entonces a la gimnasia localizada.

La gimnasia localizada, es un poco más lenta en su ritmo que la parte aeróbica, en ella se puede trabajar por bloques de ejercicios, por ejemplo, brazos, tronco y abdomen, buscando siempre un orden para no tener que estar levantando y volviendo a acostar o sentar a los practicantes en el piso, lo que hace perder tiempo de trabajo, también se trabaja de manera continuada, y si fuera necesario entre un ejercicio y otro se pueden hacer relajaciones y estiramientos. De ser posible, se pueden usar algunos implementos, dumbels, bastones, pequeños pesos, ligas, etc.

Finalizada esta parte, pasamos a la parte final de la clase ella tiene como objetivo el recuperar el organismo de los participantes buscando que su pulso baje cercano a los índices de inicio de la clase, aquí se colocan ejercicios respiratorios de recuperación y estiramientos de los músculos más utilizados durante la clase, se busca una música más suave, para calmar la excitación producida durante la clase.

\section{Conclusiones.}

A través de este trabajo logre elevar mis conocimientos acerca de la Gimnasia Aerobia y además acerca de:

- Las posiciones básicas de la Gimnasia Aerobia, así como sus combinaciones definiendo como se planifica y se realiza una sesión de clases.

- Los pasos básicos y auxiliares y sus combinaciones.

\section{Referencias bibliográficas.}

Albaladejo, L . ( 2000): Aeróbic para todos, Editorial Gymnos, Madrid .

Cagigal, J.M. (1979): Cultura Física y Cultura Integral, Editorial Kapeluz, Buenos Aires.

Carro, R. (2000): Metodología para el perfeccionamiento del Montaje de las rutinas en la Gimnasia Aeróbica Deportiva. Tesis de Maestría. (Maestría en 
Didáctica de la Educación Física Contemporánea) Universidad de Oriente.

Cooper, K. (1991): MD KlA Fitnes. A Complete Shape School, New York. (1998): Nutrición, Vida Sana y Ejercicio Físico. Conferencia, Lima. Perú.

Grupo de Desarrollo de la Educación a Distancia ISCF "Manuel Fajardo". (2006) Actividad Física en la Comunidad. [CD - ROOM. (s.1.). (s.e.).

Charola, A. (1993): Manual Práctico de Aeróbic Deportivo, Editorial Gymnos, Madrid.

Federación Internacional de Gimnasia. (2004): Código de Puntuación, FIG, Lausana, Suiza.

Federación Internacional de Gimnasia. (2004): Reglamento de competencias, FIG, Lausana, Suiza.

Navarro, D. (1996): Manual del Profesor de Educación Física, Editorial Deportes, La Habana. 


\section{PARA CITAR EL ARTÍCULO INDEXADO.}

González E., \& Días J. (2018). Gimnasia musical aerobia. Anatomía Digital, 1(3), 24-36 https://doi.org/10.33262*/visionariodigital.v3i4.949

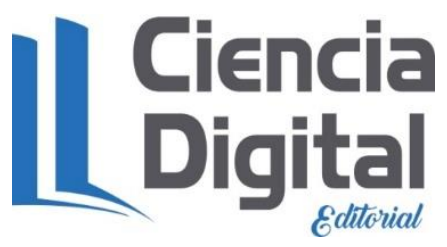

El artículo que se publica es de exclusiva responsabilidad de los autores y no necesariamente reflejan el pensamiento de la Revista Anatomía Digital.

El artículo queda en propiedad de la revista y, por tanto, su publicación parcial y/o total en otro medio tiene que ser autorizado por el director de la Revista Anatomía Digital.
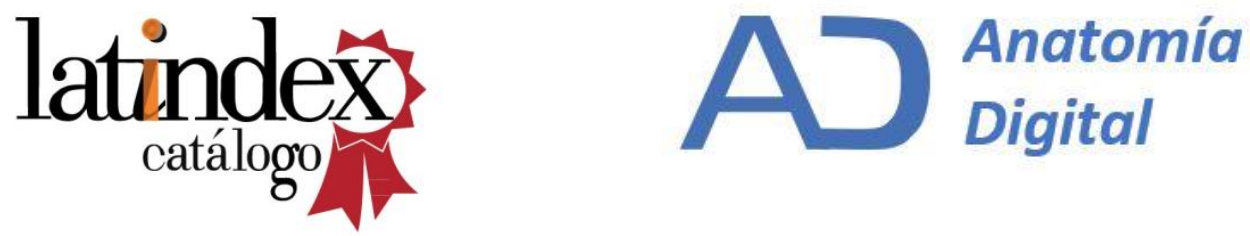